\title{
Effect of void structures in crystalline structure on the shear moduli of charged colloidal crystals
}

\author{
Linwei Wang $^{\mathrm{a}, \mathrm{b}, *}$, Shenghua $\mathrm{Xu}^{\mathrm{b}, \mathrm{c}, *}$, Hongwei Zhou $^{\mathrm{b}}$, Zhiwei Sun $^{\mathrm{b}}$, Feng Xu $^{\mathrm{a}}$ \\ a School of Civil Engineering, Beijing Jiaotong University, Beijing 100044, PR China \\ ${ }^{\mathrm{b}}$ Key Laboratory of Microgravity, Institute of Mechanics, Chinese Academy of Science, Beijing 100190, PR China \\ c School of Engineering Science, University of Chinese Academy of Sciences, Beijing 100049, PR China
}

\section{H I G H L I G H T S}

- Inconsistencies between the theoretical and experimental results for shear moduli are attributed to defects in real crystals.

- Modifications include considering the effect of void structures and using Sogami-Ise potential.

- Modified model is verified by good consistency between theoretical values and experimental results.

\section{A R T I C L E I N F O}

\section{Article history:}

Received 10 August 2016

Received in revised form

16 November 2016

Accepted 27 November 2016

Available online 28 November 2016

\section{Keywords:}

Colloidal crystals

Void defects

Shear modulus

Effective charge

Modelling

\section{G R A P H I C A L A B S T R A C T}

Shear moduli of colloidal crystals with different nearest inter-particle distances. The results show the consistency between the results of our modified model and the experimental measurements by torsional resonance spectroscopy.

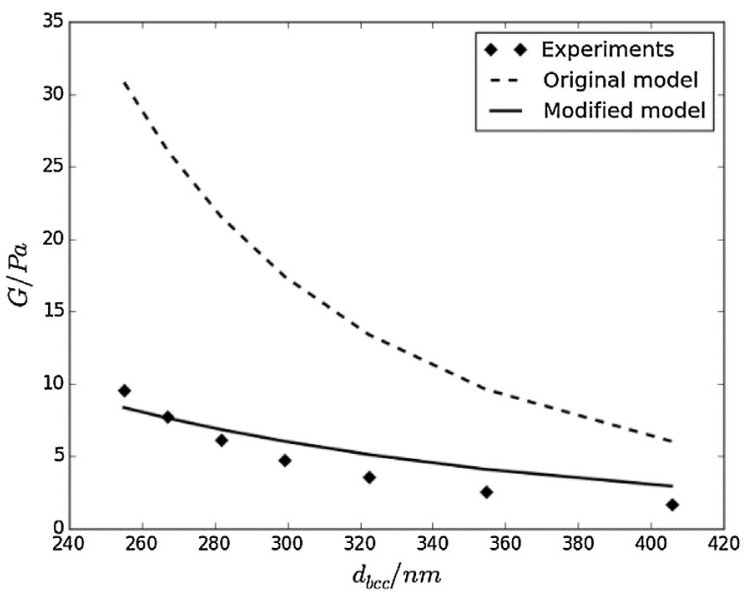

\section{A B S T R A C T}

The existing theoretical model relating shear modulus to inter-particle interaction potential always significantly overestimates the modulus compared with its experimental value for colloidal crystal formed by highly-charged particles. We suppose that such huge disagreements between the theoretical and experimental results are due to that the theoretical model is derived from perfect crystalline structures. To modify the theoretical model, the effect of void defects is introduced to the model and the SogamiIse potential model is adopted instead of the commonly used Yukawa potential model. It is shown that with these two modifications the shear modulus model produces results much more consistent with the experimental ones.

(C) 2016 Elsevier B.V. All rights reserved.

\footnotetext{
* Corresponding authors.

E-mail addresses: wanglinwei@outlook.com (L.Wang),xush@imech.ac.cn (S. Xu).
} 


\section{Introduction}

The charged colloidal particles in dispersion can self-assemble into crystalline structures under some appropriate conditions [1-3]. The formed colloidal crystals are analogous to the crystals formed by atoms and molecules and have become valuable model systems of condensed matter particularly because of their easily accessible length and time scales [4,5].

Elastic properties of materials provide an alternative approach [6-9] to understand some physical knowledge comprising the interatomic forces, structures, mechanical features, phase transitions, etc. In this respect the elasticity of the colloidal crystals has been studied in recent years both experimentally and theoretically [10-14]. Experimentally, shear elastic moduli of the colloidal crystals are usually measured through the method of torsional resonance spectroscopy (TRS) in which standing waves are excited when rotary oscillations are imposed on the colloidal crystals [15-17]. In the aspect of theoretical studies, general equations between two-body interatomic potentials in a lattice model and elastic constants were derived for monoatomic solids [18-20]. These equations were later introduced into the systems of colloidal crystals to obtain a shear modulus-potential model that describes the relationship between shear modulus of colloidal crystals and inter-particle potentials [21,22].

Actually, the two-body interaction of charged particles has been formulated with "Yukawa potential" which has a variable - the effective charge of particles. Therefore, by means of the shear modulus-potential model, the shear modulus of colloidal crystals could be estimated when the effective charge of particles in colloidal crystals is known. However, this model has never been used for colloidal crystals to directly determinate the shear modulus. It is reasonable to infer that the value of shear modulus determined by the model using the effective charge would be much larger than the experimentally measured shear modulus $G$ using TRS. It has been reported that [11], as a matter of fact, the effective shear modulus charge obtained by fitting the measured modulus using this shear modulus-potential model is always much (by about 40\%) smaller than the measured effectively transported charge.

Apparently, the theoretical model is valid now only for perfect crystals that contain no imperfections and defects. In practice, however, there are a wide variety of crystallographic defects no matter for atomic crystals or colloidal crystals [23]. Undoubtedly, structural imperfection in crystals would lower their shear moduli. For instance, it was shown [24] that in the bcc-fcc transition process of colloidal crystallization, the shear moduli are much smaller than theoretical expectations and this can be chalked up to crystalline imperfection due to not-well-equilibrated structure during the transition state. Another example demonstrating the structural imperfection is that there exist some void structures in the crystal region of crystals formed by highly-charged colloidal suspensions $[12,13,23]$.

With this regard, the aim of this study is to explore the possibilities of directly using the measured effective charge which is already known to evaluate the shear moduli based on the modified shear modulus-potential model. Previous study $[13,25]$ shows that there is structural imperfection of voids and also Sogami-Ise (SI) interaction potential is more suitable than Yukawa potential for colloidal crystals of highly-charged particles. In this study these two factors were taken into account as modifications to the shear moduluspotential model. The modified model was verified by comparing the theoretical estimation of shear moduli with experimental values measured by TRS. Our results show that the shear moduli evaluated from the modified model are quite consistent with the measured values when the effectively transported charges are used inside the pair-potential model.

\section{Theory}

The shear moduli $G$ expressed in the original shear moduluspotential model for perfect colloidal crystals with body-centered cubic (b.c.c.) and face-centered cubic (f.c.c.) structures have the following formulas $[11,16,22]$ :

$G_{b c c}\left(d_{b c c}\right)=f_{A} \frac{3 \sqrt{3}}{4 d_{b c c}^{3}}\left(\left.\frac{4}{9} d_{b c c}{ }^{2} \frac{\partial^{2} V(r)}{\partial r^{2}}\right|_{r=d_{b c c}}+\left.\frac{8}{9} d_{b c c} \frac{\partial V(r)}{\partial r}\right|_{r=d_{b c c}}\right)(1)$

and

$G_{f c c}\left(d_{f c c}\right)=f_{A} \frac{\sqrt{2}}{d_{f c c}{ }^{3}}\left(\left.\frac{1}{2} d_{f c c}{ }^{2} \frac{\partial^{2} V(r)}{\partial r^{2}}\right|_{r=d_{f c c}}+\left.\frac{3}{2} d_{f c c} \frac{\partial V(r)}{\partial r}\right|_{r=d_{f c c}}\right)$

where $d_{b c c}, d_{f c c}$ are the nearest inter-particle distance for b.c.c. and f.c.c. sturctures, respectively, $V(r)$ is the effective inter-particle interaction pair-potential with $r$ to be the distance between two particles, and $f_{A}$ is a numerical factor that accounts for the different boundary conditions in averaging over randomly oriented crystallites or local environs. Its theoretical limits are $f_{A}=0.4$ for homogeneously distributed strains and $f_{A}=0.6$ for homogeneously distributed stresses, respectively [26,27]. For polycrystalline samples a value of $f_{A}=0.5$ is encountered in most cases $[16,28]$ and will also be used in this work.

The Yukawa potential $V_{\text {Yukawa }}(r)$ which considers Coulomb repulsions only was usually used for $V(r)$ in this shear modulus-potential model before $[11,29,30]$. However, experimental observations indicated that there are voids appeared in highly-charged colloidal crystals $[12,31,32]$. So in addition to the repulsion, inclusion of the conterions-mediated long-range attraction was suggested and then the inter-particle interaction potential was replaced by SI potential [33,34]. The SI potential model, based on the Gibbs free energy of the interaction, not only take into account the large size difference between colloidal particles and small ions, but also relates the variation of macroion charge to the release of counterions. The expressions of Yukawa [11,15,35] and SI pair-potential energy [36,37] are:

$V_{\text {Yukawa }}(r)=\frac{Z_{e}^{2} e^{2}}{4 \pi \varepsilon_{0} \varepsilon_{r}}\left[\frac{\exp (\kappa a)}{1+\kappa a}\right]^{2} \frac{\exp (-\kappa r)}{r}$

and

$V_{S I}(r)=\frac{Z_{e}^{2} e^{2}}{4 \pi \varepsilon_{0} \varepsilon_{r}}\left[\frac{\sinh (\kappa a)}{\kappa a}\right]^{2}\left(\frac{A}{r}-\frac{\kappa}{2}\right) \exp (-\kappa r)$

where $Z_{e}$ is the effective charge number of particles, $e$ is the elementary charge, $r$ is the inter-particle distance, $a$ is the radius of particles, and $A=1+(\kappa a) \operatorname{coth}(\kappa a)[25,36]$. The parameter $\kappa$ is the inverse Debye-screening length given by $[15,38]$ :

$\kappa^{2}=\frac{e^{2}}{\varepsilon_{0} \varepsilon_{r} k_{B} T}\left(n Z_{e}+n_{\text {salt }}\right)$

where $n$ is the particle number density of the suspension, $\varepsilon_{0} \varepsilon_{r}$ the dielectric permittivity of the suspension, $k_{B} T$ the thermal energy, and $n_{\text {salt }}=2000 N_{A} c[12,15]$ the number density of background electrolyte with $N_{A}$ being Avogadro constant and $c$ the molar concentration of foreign salt $\left(\mathrm{mol} \mathrm{L}^{-1}\right)$.

It is obvious that the expressions of shear modulus-potential model shown in Eq. (1) and (2) do not take into account the effect of voids in crystalline structure and they are valid only for perfect crystals. In order to improve this situation there are two factors that can be taken to modify this model: factor 1: taking into account the void defects in crystalline structure; factor 2: inclusion of the attraction in interaction potential model.

For the first factor, we considered that the micro-structure of colloidal crystals is similar to that of cellular solids whose elastic properties have been studied thoroughly for a long time [39-41]. 
The single most important structural characteristic of a cellular solid is its relative density $\left(\rho / \rho_{0}\right)$, where $\rho$ is the average density of the cellular material as a whole and $\rho_{0}$ is the density of the components alone. Moreover, Gibson-Ashby model [42,43] shows that cellular solid Young's modulus is proportional to the relative density raised to the second power, but Poisson's ratio has no correlation with it. We can use the similar approach in treating cellular solids to deal with colloidal crystals containing voids inside. To do so, we need to find a parameter that is equivalent to $\left(\rho / \rho_{0}\right)$. First of all, we use $d_{u n i}$ to denote the average nearest inter-particle distance when crystals formed uniformly by colloidal particles fill the entire volume of their container without any void inside. On the other hand, if there are voids in the crystals this distance will be smaller than $d_{u n i}$ and it can be experimentally determined by the reflection spectrum method, and therefore we denote it by $d_{\exp }$ here. Now we can see that $\left(d_{\exp } / d_{u n i}\right)^{3}$ for colloidal crystals is equivalent to the relative density $\left(\rho / \rho_{0}\right)$. Since a part of volume is occupied by voids [12], $d_{\text {exp }}<d_{\text {uni }}$. The volume percentage of voids [44] is equal to $1-\left(d_{\text {exp }} / d_{\text {uni }}\right)^{3}$.

To consider the effect of void structures, now we introduce a prefactor $\left(d_{\text {exp }} / d_{u n i}\right)^{6}$ to represent the modulus of colloidal crystals with void structure inside. Then we can update the shear modulus model of colloidal crystal bulk with entire volume as:

$$
\begin{aligned}
& G_{b c c}\left(d_{b c c}\right)=f_{A} \frac{3 \sqrt{3}}{4 d_{b c c}{ }^{3}}\left(\frac{d_{\text {exp }}}{d_{u n i}}\right)^{6}\left(\left.\frac{4}{9} d_{b c c}{ }^{2} \frac{\partial^{2} V(r)}{\partial r^{2}}\right|_{r=d_{b c c}}\right. \\
& \left.+\left.\frac{8}{9} d_{b c c} \frac{\partial V(r)}{\partial r}\right|_{r=d_{b c c}}\right)
\end{aligned}
$$

and

$$
\begin{aligned}
& G_{f c c}\left(d_{f c c}\right)=f_{A} \frac{\sqrt{2}}{d_{f c c}{ }^{3}}\left(\frac{d_{\text {exp }}}{d_{u n i}}\right)^{6}\left(\left.\frac{1}{2} d_{f c c}{ }^{2} \frac{\partial^{2} V(r)}{\partial r^{2}}\right|_{r=d_{f c c}}\right. \\
& \left.+\left.\frac{3}{2} d_{f c c} \frac{\partial V(r)}{\partial r}\right|_{r=d_{f c c}}\right) .
\end{aligned}
$$

Since the sixth power of $d_{\text {exp }} / d_{u n i}$ is presented in Eq. (6) and (7), the influence of voids would not be ignorable generally even when $d_{\text {exp }} / d_{\text {uni }}$ is close to 1 .

For the second factor to improve the existing shear moduluspotential model, SI potential, Eq. (4), shall be used instead of Yukawa potential, Eq. (3), in the shear modulus-potential model.

In charged colloidal suspensions, the electrostatic coupling between oppositely charged species induces a strong accumulation of counterions in the vicinity of the macroion surface. This counterion condensation, which accounts for screening of the intrinsic particle charge, causes a colloidal particle to have an effective charge which is much less than the actual bare charge. When calculating the theoretical value of the effective inter-particle interaction pair-potential model, the effectively transported charge $Z_{\sigma}^{*}$ is used here.

\section{Material and methods}

\subsection{Preparation and characterization of samples}

The negatively charged polystyrene (PS) particles used here in experiments were synthesized by an emulsion polymerization method [45]. Filtration and centrifugation were employed to ensure the monodispersity of the yielding particles. Meanwhile, PS particles were purified by repeated washing with deionized water (UPRLC5DRO UPW system, Relatec, Beijing). Thereafter PS particles were stored with resin (Amberlite IRN-150, EMD Millipore Corporation, Germany) for the sake of further deionization. The mean
Table 1

Parameters of colloidal particles.

\begin{tabular}{lllll}
\hline Particle & $2 a / \mathrm{nm}$ & PDI & $10^{8} \mu_{p} /\left(\mathrm{m}^{2} \mathrm{~V}^{-1} \mathrm{~s}^{-1}\right)$ & $Z_{\sigma}^{*}$ \\
\hline PS-1 & 81 & 0.02 & 4.3 & 668 \\
PS-2 & 82 & 0.05 & 5.1 & 650 \\
PS-3 & 86 & 0.05 & 5.1 & 705 \\
PS-4 & 83 & 0.02 & 4.3 & 736 \\
\hline
\end{tabular}

$2 a$ is the average diameter, PDI is the polydispersity index to describe the monodispersity of particles [46], $\mu_{p}$ is the electrical mobility of particles in deionized water, $Z_{\sigma}^{*}$ is the effectvely transported charge of particles.

diameter and polydispersity index of particles used in this work were measured by dynamic light scattering(BI-200SM, Brookhaven Instruments Corporation, USA), as shown in Table 1.

The setup of reflection spectroscopy used to measure the nearest inter-particle distance of colloidal crystals is composed of a fiber optic spectrometer(Avaspec-2048, Avantes, Netherland) with a tungsten halogen light source (Avalight-HAL, Avantes, Netherlands) and a bifurcated fiber optic cable. In the reflection measurements, the incident light hits the surface of the sample at right angles and the light is turned back by Bragg diffraction. This corresponds to the reflection spectrum. For both b.c.c. and f.c.c. structure the distance at a scattering angle of $90^{\circ}$ is given by $[47,48]$ :

$d_{\exp }=0.6124 \lambda / v$,

where $v$ is the refractive index of the colloidal dispersion (taken that of water in this work) and $\lambda$ is the peak wavelength for the refractive intensity.

\subsection{Effectively transported charge measurement}

In the case of deionized dispersions, assuming the additivity of all conductivity contributions, the conductivity of the suspension is well described by $[49,50]$ :

$\sigma=\sigma_{0}+\sigma_{B}=n e Z_{\sigma}^{*}\left(\mu_{p}+\mu_{H^{+}}\right)+\sigma_{B}$,

where $\sigma_{0}$ is the conductivity of PS particles and counterions, $\sigma_{B}$ is the background conductivity, $n$ is the number density of PS particles and $e$ is the elementary charge, $\mu_{p}$ and $\mu_{H^{+}}$are the mobility of charged particles and protons, respectively. The value of $\mu_{H^{+}}$ is $36.5 \times 10^{-8} \mathrm{~m}^{2} \mathrm{~V}^{-1} \mathrm{~s}^{-1}$ [51] and $\mu_{p}$ can be measured by Laser Doppler Velocimety (ZetaPlus, Brookhaven Instruments corporation, USA) in the laboratory [52] as shown in Table 1. Giving that the conductivity $\sigma$ increases linearly with increasing particle number density $n$, the effectively transported charge $Z_{\sigma}^{*}$ is taken as the only free fitting parameter in the relationship of Eq. (9). An example measurement is given for PS-1 in Fig. 1, and the results for all particles of this work are listed in Table 1.

\subsection{Shear modulus determination}

Shearing elasticity of colloidal crystal samples was probed by TRS [17]. The cylindrical sample cell was put into oscillations with very low amplitude around its vertical axis. As the shear modulus of the sample are small, standing waves are excited at wavelengths which are comparable to the cell dimensions [53]. This situation corresponds to harmonic lattice vibrations on the colloidal level which can be visible as shifts of the Bragg-peaks or distortions of the first structure factor peak. These standing waves are detected and frequency analyzed by time resolved static light scattering in combination with lock-in technique $[16,21,54]$. Resonance frequencies are observed at $[15,21]$ :

$f_{j m}^{2}=\frac{G\left[\mu_{j}^{2}+(m+1)^{2} \pi^{2} \alpha^{2}\right]}{(2 \pi)^{2} \rho R^{2}}$, 


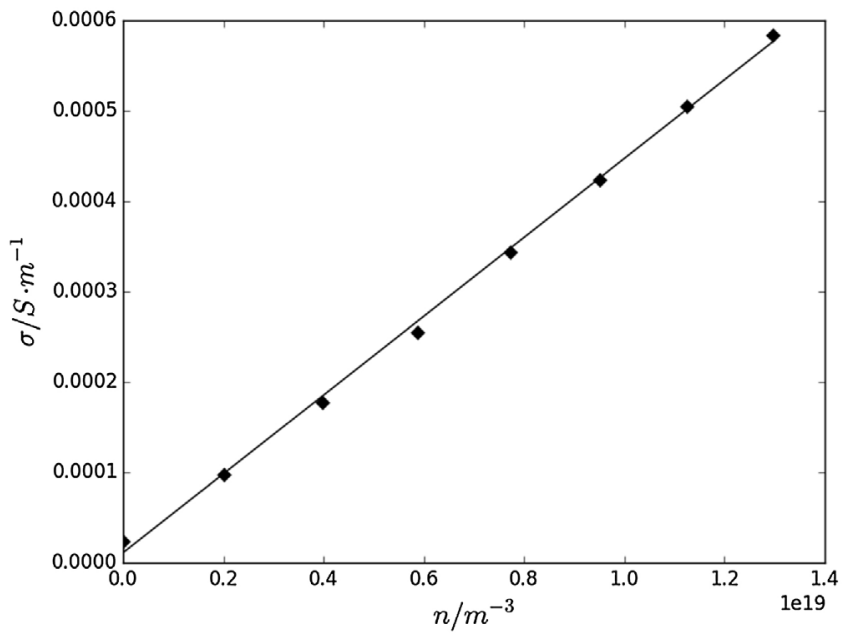

Fig. 1. The conductivity of the suspension as a function of particle number density $n$ for particles PS-1. A constant effectively transported charge of $Z_{\sigma}^{*}=668$ is derived.
Table 2

The ratio $d_{\text {exp }} / d_{u n i}$ for the different types of particles.

\begin{tabular}{lllll}
\hline Particle & PS-1 & PS-2 & PS-3 & PS-4 \\
\hline$d_{\text {exp }} / d_{\text {uni }}$ & 0.93 & 0.95 & 0.89 & 0.88 \\
\hline
\end{tabular}

where $j$ and $m$ are the indices of the order of the resonances, $\alpha$ is the ratio between the cell radius $R$ and the filling height $H, \rho$ is the mass density of the colloidal dispersions, and $\mu_{j}$ the zeroes of the first order Bessel function $J_{1}$. Then the shear modulus $G$ can be derived through this equation.

\section{Results and discussion}

In the present study four kinds of colloidal particles are prepared. All the values of $d_{\exp }$ were determined by the measurement of reflection spectroscopy and those of $d_{\text {uni }}$ were theoretically calculated according to the volume fraction of colloidal particles. The ratios $d_{\text {exp }} / d_{u n i}$ of colloidal crystals formed by these particles are shown in Table 2 . And their shear moduli were experimentally measured by TRS as shown in Fig. 2 and were also compared with theoretical results. In this work, all the formed colloidal crystals are b.c.c. structures.

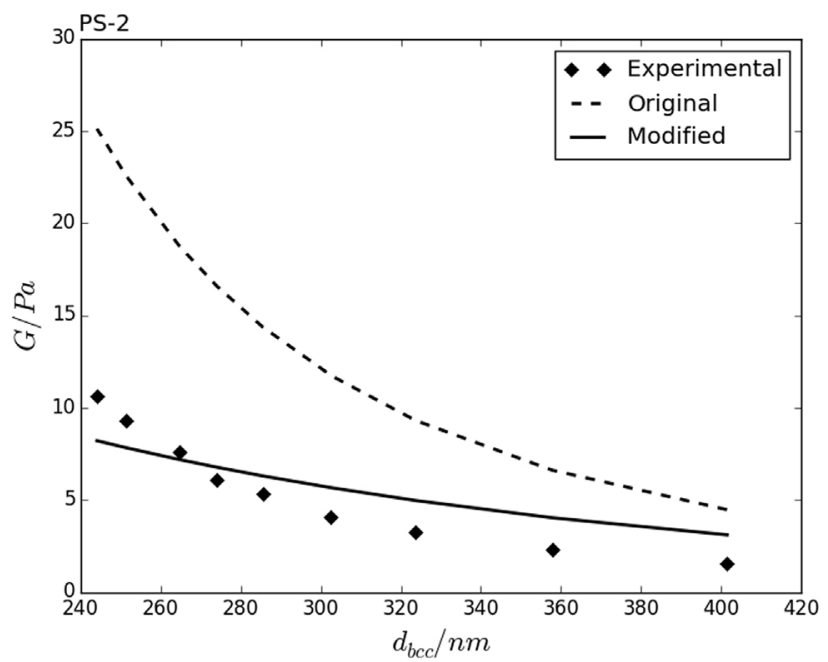

(b) PS-2

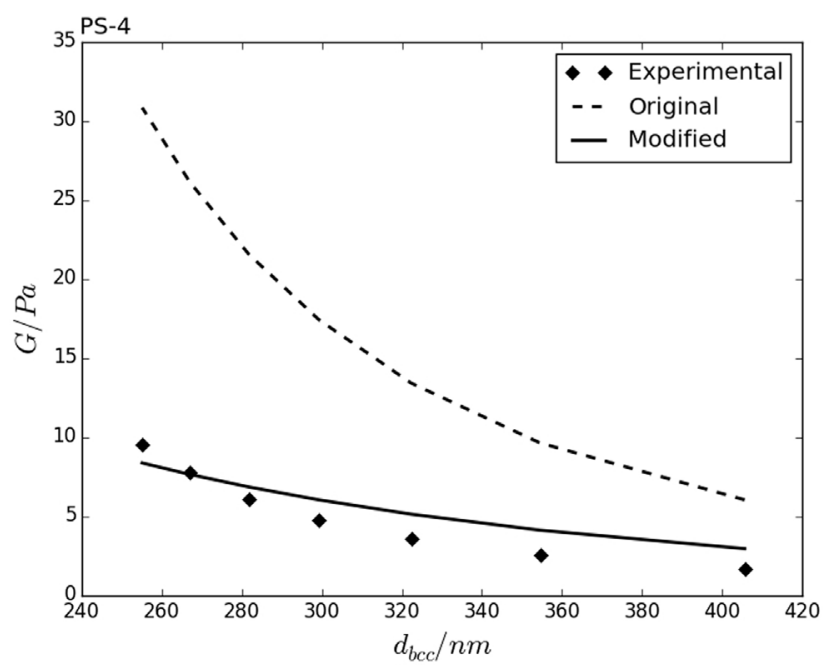

(d) PS-4

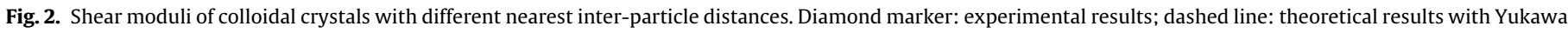
potential used and no void defects considered; solid line: theoretical results with SI potential used and considered void defects. 
The effectively transported charge $Z_{\sigma}^{*}$ is used for $Z_{e}$ in their expressions. Besides, a constant background concentration of $2 \times$ $10^{7} \mathrm{~mol} \mathrm{~L}^{-1}$ of dissociated carboxylic acid is assumed according to previous papers $[11,15]$.

From Fig. 2 it demonstrates that theoretical values in the original model without modification produces larger deviation from the experimental results. Comparatively, the data evaluated in the modified model are much better coincident with experimental values. The results show that the two factors introduced in the modified model are necessary. It should be mentioned here that the value of $d_{\text {exp }} / d_{\text {uni }}$ is 0.95 for PS-2, which is quite close to 1 . Nevertheless, the influence of voids is still needed to be considered because the effects of voids are proportional to the six order of $d_{\text {exp }} / d_{\text {uni }}$, which is about 0.735 and thus cannot be neglected.

In addition, for colloidal crystals formed by the same kind of particles, it is obvious that the deviations between the original theoretical predictions and experimental results increase as the decreases of nearest inter-particle distance. However, we found the deviations can be tolerable in the whole range of inter-particle distance after modification. So the smaller the nearest inter-particle distance, the greater will be the influence of void defect to the shearing elasticity. It is because that the difference by using SI and Yukawa potential is not so obvious when the nearest neighbor distance is large. By comparing the original model line and the modified model line, it can also be found that the smaller the nearest inter-particle distance, the more remarkable will be the improvement by the two modifications.

\section{Conclusions}

In the course of the present work, a modified theoretical model of shear modulus of elasticity for colloidal crystals is proposed in order to introduce the influence of void structures inside and use a proper inter-particle interaction potential. Theoretical values of the original and the modified model are compared with experimental results for the colloidal crystals formed by four kinds of particles. It can be concluded that the theoretical results of the shear modulus-potential model considered the influence of void structures and based on SI pair-potential is able to describe the experimental results better than the model not considered the influence of void structures and based on Yukawa pair-potential. Therefore we can now estimate the practical value of shear moduli of colloidal crystals theoretically.

There are various types of imperfections and defects that affect the mechanical properties in the colloidal crystals, however only the void structures are considered in this work. Consequently, the effective shear modulus charge of particles we got here is still small compared to the effectively transported charge. Further improvements for the theoretical model might be possible, for instance, to consider the influences of additional imperfections or defects, such as the heterogeneity of crystallites size and the irregularity of grain orientation.

\section{Acknowledgement}

We gratefully acknowledge financial support by grants 11302226,11572322 and 11672295 from the National Natural Science Foundation of China.

\section{References}

[1] W.B. Russel, Condensed-matter physics: tunable colloidal crystals, Nature 421 (2003) 490-491

[2] V.J. Anderson, H.N.W. Lekkerkerker, Insights into phase transition kinetics from colloid science, Nature 416 (2002) 811-815.
[3] J. Luo, D. Qu, A. Tikhonov, J. Bohn, S.A. Asher, Monodisperse high refractive index, highly charged ZnS colloids self assemble into crystalline colloidal arrays, J. Colloid Interface Sci. 345 (2010) 131-137.

[4] A. Yethiraj, Tunable colloids: control of colloidal phase transitions with tunable interactions, Soft Matter 3 (2007) 1099-1115

[5] P.J. Yunke, M.D. Gratale, M.A. Lohr, T. Still, A.G. Yodh, Physics in ordered and disordered colloidal matter composed of poly( $\mathrm{N}$-isopropylacrylamide) microgel particles, Rep. Prog. Phys. 77 (2014) 056601.

[6] P. Wette, H.J. Schöpe, J. Liu, T. Palberg, Characterisation of Colloidal Solids, Trends in Colloid and Interface Science, vol. XVI, Springer, 2004, pp. 264-268.

[7] D. Anderson, D. Schaar, H.G.E. Hentschel, J. Hay, P. Habdas, E.R. Weeks, Local elastic response measured near the colloidal glass transition, J. Chem. Phys. 138 (2013) 12A520.

[8] G. Meng, J. Paulose, D.R. Nelson, V.N. Manoharan, Elastic instability of a crystal growing on a curved surface, Science 343 (2014) 634-637.

[9] W. Kung, P. Ziherl, R.D. Kamien, The foam analogy: from phases to elasticity, J. Colloid Interface Sci. 275 (2004) 539-547.

[10] R.S. Crandall, R. Williams, Gravitational compression of crystallized suspensions of polystyrene spheres, Science 198 (1977) 293-295.

[11] P. Wette, H.J. Schöpe, T. Palberg, Comparison of colloidal effective charges from different experiments, J. Chem. Phys. 116 (2002) 10981-10988.

[12] H. Zhou, S. Xu, W. Ouyang, Z. Sun, L. Liu, Influence of the surface charge on the homogeneity of colloidal crystals, J. Chem. Phys. 139 (2013) 064904.

[13] W. Ouyang, H. Zhou, S. Xu, Z. Sun, Molecular dynamics study of homogeneous and inhomogeneous phase in charged colloids: the influence of surface charge density, Colloid Surf. A 441 (2014) 598-605.

[14] D. Tang, A.G. Marangoni, Modified fractal model and rheological properties of colloidal networks, J. Colloid Interface Sci. 318 (2008) 202-209.

[15] P. Wette, H.J. Schöpe, T. Palberg, Experimental determination of effective charges in aqueous suspensions of colloidal spheres, Colloid. Surf. A 222 (2003) 311-321.

[16] H. Zhou, S. Xu, Z. Sun, R. Zhu, Shear moduli in bcc-fcc structure transition of colloidal crystals, J. Chem. Phys. 143 (2015) 144903

[17] H.-J. Schöpe, T. Palberg, A multipurpose instrument to measure the vitreous properties of charged colloidal solids, J. Colloid Interface Sci. 234 (2001) $149-161$

[18] K. Fuchs, A quantum mechanical calculation of the elastic constants of monovalent metals, Proc. R. Soc. A 53 (1936) 622-639.

[19] K. Fuchs, The elastic constants and specific heats of the Alkali Metals, Proc. R. Soc. A 57 (1936) 444-450.

[20] R.A. Johnson, Relationship between two-body interatomic potentials in a lattice model and elastic constants, Phys. Rev. B 6 (1972) 2094-2100.

[21] E. Dubois-Violette, P. Pieranski, F. Rothen, L. Strzelecki, Shear waves in colloidal crystals: i. Determination of the elastic modulus, J. Phys. France 41 (1980) 369-376.

[22] J.F. Joanny, Acoustic shear waves in colloida crystals, J. Colloid Interface Sci. 71 (1979) 622-624

[23] H. Yoshida, K. Ito, N. Ise, Localized ordered structure in polymer latex suspensions as studied by a confocal laser scanning microscope, Phys. Rev. B 44 (1991) 435-438.

[24] H. Zhou, S. Xu, Z. Sun, X. Du, L. Liu, Kinetics study of crystallization with the disorder-bcc-fcc phase transition of charged colloidal dispersions, Langmuir 27 (2011) 7439-7445.

[25] B.V.R. Tara, R.G. Joshi, I.S. Sogami, Long-range attraction between like-charged colloids: simulations and microgravity experiments, Int. J. Microgravity Sci. Appl. 32 (2015) 320209.

[26] Z. Hashin, S. Shtrikman, A variational approach to the theory of the elastic behaviour of polycrystals, J. Mech. Phys. Solids 10 (1962) 343-352.

[27] R. Zeller, P.H. Dederichs, Elastic constants of polycrystals, Phys. Status Solidi B 55 (1973) 831-842

[28] H.J. Schöpe, T. Decker, T. Palberg, Response of the elastic properties of colloidal crystals to phase transitions and morphological changes, J. Chem. Phys. 109 (1998) 10068-10074.

[29] C. Desgranges, J. Delhommelle, Polymorph selection during the crystallization of Yukawa systems, J. Chem. Phys. 126 (2007) 054501.

[30] C.P. Royal, M.E. Leunissen, A. van Blaaderen, A new colloidal model system to study long-range interactions quantitatively in real space, J. Phys.-Condens. Matter 15 (2003) S3581-S3596.

[31] H. Yoshida, N. Ise, T. Hashimoto, Void structure and vapor-liquid condensation in dilute deionized colloidal dispersions, J. Chem. Phys. 103 (1995) 10146-10151.

[32] H. Yoshida, J. Yamanaka, T. Koga, T. Koga, N. Ise, T. Hashimoto, Transitions between ordered and disordered phases and their coexistence in dilute ionic colloidal dispersions, Langmuir 15 (1999) 2684-2702.

[33] N. Ise, T. Konishi, B.V.R. Tata, How homogeneous are homogeneous dispersions? counterion-mediated attraction between like-charged species, Langmuir 15 (1999) 4176-4184.

[34] B.V.R. Tata, N. Ise, Homogeneous to inhomogeneous transition in charged colloids, Phys. Rev. B 54 (1996) 6050-6053.

[35] M.J. Stevens, M.L. Falk, M.O. Robbins, Interactions between charged spherical macroions, J. Chem. Phys. 104 (1996) 5209-5219.

[36] I. Sogami, N. Ise, On the electrostatic interaction in macroionic solutions, J Chem. Phys. 81 (1984) 6320-6332.

[37] B.V.R. Tata, N. Ise, Monte Carlo study of structural ordering in charged colloids using a long-range attractive interaction, Phys. Rev. E 58 (1998) 2237-2246. 
[38] T. Okubo, Importance of electrical double layers in structural and diffusional properties of deionized colloidal suspensions, Colloid Surf. A 109 (1996) 77-88.

[39] V.J. Anderson, E.M. Terentjev, S.P. Meeker, J. Crain, W.C.K. Poon, Cellular solid behaviour of liquid crystal colloids 1. Phase separation and morphology, Eur. Phys. J. E 4 (2001) 11-20.

[40] V.J. Anderson, E.M. Terentjev, Cellular solid behaviour of liquid crystal colloids 2. Mechanical properties, Eur. Phys. J. E 4 (2001) 21-28.

[41] M.F. Ashby, R.F.M. Medalist, The mechanical properties of cellular solids, Metall. Trans. A 14 (1983) 1755-1769.

[42] L.J. Gibson, M.F. Ashby, Cellular Solids: Structures and Properties, Cambridge University Press, United Kingdom, 1997.

[43] T.G. Nieh, J.H. Kinney, J. Wadsworth, A.J.C. Ladd, Morphology and elastic properties of aluminum foams prodeced by a casting technique, Scr. Mater. 38 (1998) 1487-1494.

[44] S.B. Debord, L.A. Lyon, Influence of particle volume fraction on packing in responsive hydrogel colloidal crystals, J. Phys. Chem. B 107 (2003) 2927-2932.

[45] X. Du, S.-h. Xu, Z.-W. Sun, L. Liu, Influence of sedimentation on crystallization of charged colloidal particles, Chin. J. Chem. Phys. 25 (2012) 318-324.

[46] B. Siekmann, H. Bunjes, M.H.J. Koch, K. Westesen, Preparation and structural investigations of colloidal dispersions prepared from cubic monoglyceride water phases, Int. J. Pharm. 244 (2002) 33-43.
[47] T. Okubo, Elastic modulus of crystal-like structures of deionized colloidal spheres in sedimentation equilibrium as studied by the reflection-spectrum method, J. Chem. Soc. Faraday Trans. 1 (1989) 455-466.

[48] H. Zhou, S. Xu, Z. Sun, X. Du, J. Xie, Rapid determination of colloidal crystal's structure by reflection spectrum, Colloid Surf. A 375 (2011) 50-54.

[49] Y.-K. Gong, K. Nakashima, R. Xu, A novel method to determine effective charge of polystyrene latex particles in aqueous dispersion, Langmuir 17 (2001) 2889-2892.

[50] D. Hessinger, M. Evers, T. Palberg, Independent ion migration in suspensions of strongly interacting charged colloidal spheres, Phys. Rev. E 61 (2000) 5493-5506.

[51] R.N. Varney, Mobility of hydrogen ions, Phys. Rev. Lett. 5 (1960) 559-560.

[52] C.P. Wang, Laser doppler velocimetry, J. Quant. Spectrosc. Radiat. 40 (1988) 309-319.

[53] H.M. Lindsay, P.M. Chaikin, Elastic properties of colloidal crystals and glasses, J. Chem. Phys. 76 (1982) 3774-3781.

[54] M. Joanicot, M. Jorand, P. Piéranski, F. Rothen, Shear waves in colloidal crystals: II. Effects of finite height in cylindrical samples, J. Phys. France 45 (1984) 1413-1421. 